\title{
Episodio de hipotonía-hiporreactividad posvacunación con DTPa
}

\author{
Hypotonic-hyporesponsive episode following acellular pertussis vaccination
}

\author{
Lic. Olga Díaz Morales ${ }^{a}$ Lic. José David Martinez-Pajares ${ }^{a}$, Lic. Juan Trigo Moreno ${ }^{a}$, Lic. Juan Carlos Ramos Díaz ${ }^{a}$
}

\begin{abstract}
RESUMEN
La introducción de la vacunación sistemática contra la tos ferina determinó un descenso drástico en la incidencia de esta patología, aunque todavía representa un problema de salud pública, incluso en los países con alta cobertura de vacunación. Sin embargo, además de la disminución de los casos por la profilaxis activa, se ha observado la emergencia de efectos adversos neurológicos graves, como los denominados "episodios de hipotonía-hiporreactividad" (HHE), caracterizados por una pérdida súbita del tono muscular asociada a escasa respuesta a los estímulos y palidez cutánea o cianosis.

Los HHE son un fenómeno infrecuente después de la administración de la vacuna con un componente antitosferínico, pero es fundamental que el personal sanitario sepa reconocerlos, sobre todo en las siguientes 48 horas de la vacunación. Aunque en ocasiones es difícil establecer una relación causal entre la administración de la vacuna y un efecto adverso, debe comunicarse cualquier evento sospechoso.

Palabras clave: tos ferina, vacuna, episodio de hipotonía-hiporreactividad.
\end{abstract}

\begin{abstract}
The introduction of routine vaccination against whooping cough caused a drastic decline in the incidence of this disease, but remains today a public health problem even in countries with high vaccination coverage. However, with this decrease in cases, there were an emergence of neurological severe adverse events such as the "hypotonic-hyporesponsive episodes" (HHE), characterized by sudden loss of muscle tone associated with poor response to stimuli and skin pallor or cyanosis. The HHE is a rare phenomenon after administration of pertussis vaccine, but it is essential for health workers recognition of such reactions, especially in the 48 hours following vaccination. Although sometimes can become difficult to attribute a causal relationship between vaccine administration and an adverse effect, any suspicious events should be reported.

Keywords: whooping cough; vaccine; hypotonic-hyporesponsive episode.
\end{abstract}

http:/ /dx.doi.org/10.5546/aap.2013.e97

a. Unidad de Gestión Clínica de Pediatría.

Hospital General de Antequera, Málaga, España.

Correspondencia:

Lic. Olga Díaz Morales: olga.dimo@hotmail.com

Conflicto de intereses: Ninguno que declarar.

Recibido: 26-9-12

Aceptado: 6-5-13

\section{INTRODUCCIÓN}

Los dos últimos siglos han demostrado ampliamente que las vacunas son eficaces y que las estrategias para su uso han supuesto un beneficio para la salud de los niños y la potencial erradicación de enfermedades con reservorio humano exclusivo, como ya ocurrió con la viruela. Pero aunque la eficacia de las vacunas sigue siendo un pilar fundamental, la seguridad ha adquirido un papel cada vez más destacado. Por ello, el conocimiento y la identificación de los efectos adversos supuestamente atribuibles a las vacunas cobran cada día mayor interés.

Con el inicio del uso de la vacuna celular contra la tos ferina (DTPw), en la década de 1940, se obtuvo un descenso importante en la incidencia de esta patología pero, a su vez, surgieron numerosos efectos secundarios neurológicos, como los denominados "episodios de hipotoníahiporreactividad" (HHE), caracterizados por la pérdida súbita del tono muscular asociada a una escasa respuesta a los estímulos y palidez cutánea o cianosis.

Si bien la introducción de las vacunas acelulares (DTPa, dTpa) ha reducido la incidencia de dichos episodios, ${ }^{1}$ todavía se muestra como un efecto adverso para tener en cuenta. Se presenta el caso de un lactante con sospecha inicial de shock séptico, que finalmente resultó ser un HHE.

\section{CASO CLÍNICO}

Un lactante de 2 meses, sin antecedentes de interés y con un estado de vacunación según el calendario (primera dosis de HBV), ${ }^{2}$ después de 8 horas de recibir la vacuna correspondiente a su edad en el subsector privado (vacuna séxtuple [DTPa, Hib, IPV, HB] y meningocócica C), fue llevado a la consulta al Servicio de Urgencias por la instauración brusca de palidez cutánea acompañada de hipotonía e hiporreactividad. En el examen se destacaba un regular estado general, con cianosis y frialdad de las extremidades, así como hipotonía y escasa respuesta a los estímulos. Las constantes vitales (frecuencia cardíaca, frecuencia respiratoria, tensión arterial) y la saturación de oxígeno respirando aire ambiente se 
encontraban en rangos normales; la temperatura rectal era de $38,6^{\circ} \mathrm{C}$.

Ante la sospecha inicial de un cuadro séptico, se inició expansión volumétrica y, después de practicar un estudio analítico y de obtener muestras para cultivo, se comenzó con el tratamiento antibiótico empírico. En las dos horas sucesivas al ingreso, presentó una mejoría clínica llamativa, con recuperación del tono muscular y reactividad a los estímulos normales, así como buena perfusión tisular, observándose un lactante asintomático y con examen físico normal. Tanto el despistaje infeccioso inicial como los diversos cultivos obtenidos de la sangre, la orina y el líquido cefalorraquídeo resultaron negativos, por lo que se replanteó el diagnóstico inicial de sepsis y se atribuyó el episodio a un posible efecto adverso de la vacuna acelular contra la tos ferina.

Siguiendo las recomendaciones del Comité de Enfermedades Infecciosas de la Academia Estadounidense de Pediatría y, ante la ausencia de contraindicaciones absolutas, se administró la dosis correspondiente a la vacunación de los 4 meses bajo observación hospitalaria, sin que se presentaran manifestaciones clínicas en las 48 horas siguientes.

\section{COMENTARIO}

La tos ferina sigue siendo un problema de salud pública. Según los datos provenientes de la Organización Mundial de la Salud, en 2008 se estimaron 16 millones de casos en el mundo con 195000 muertes, sobre todo en los países en desarrollo. ${ }^{3}$

La introducción de la vacunación sistemática con vacunas de células enteras en la década de 1940 fue determinante para el descenso de su incidencia, aunque todavía sigue siendo la enfermedad inmunoprevenible menos controlada en los países industrializados, ${ }^{3}$ debido, en parte, a la pérdida de la inmunidad posvacunación a partir de los 3 a 5 años de la última dosis. Sin embargo, paralelamente a esta disminución de la incidencia, se describió la emergencia de efectos adversos neurológicos, como crisis convulsivas $(13,4 / 1000000),{ }^{4}$ espasmos $(0,39 / 1000000),{ }^{4}$ encefalopatía posvacunal $(0,78 / 1000000){ }^{4}$ o los denominados "episodios de hipotoníahiporreactividad" (7-71/100 000). ${ }^{5}$

La encefalopatía se asocia a los niños que inician una regresión psicomotora y crisis convulsivas tras la administración de la vacuna, particularmente con Bordetella pertussis inactivada. Durante décadas se consideró que existía una relación causal entre ambas, pero recientemente se ha descrito que muchos de esos pacientes fueron más tarde diagnosticados de síndrome de Dravet o de Angelman, con lo cual se comenzó a sugerir que la vacunación no era una causa sino un posible desencadenante en pacientes genéticamente predispuestos. ${ }^{6}$

Los HHE se describieron por primera vez hace tres décadas, si bien existían publicaciones previas en las que se detallaban fenómenos similares, conocidos como "colapso" o "shock". 1,5,7 Esta diversidad en la definición justificaría la variación de la incidencia entre los diferentes estudios; por ello, y con el objetivo de unificar los términos referidos a un mismo evento, en 1998 se estableció la descripción de caso de HHE (Tabla 1). ${ }^{8}$ Suelen ocurrir después de la vacunación con componentes contra la tos ferina, pero también se describieron asociados a otras, como hepatitis B y Haemophilus influenzae de tipo $b .{ }^{5}$

$\mathrm{Su}$ mecanismo fisiopatológico no es bien conocido y pueden estar involucrados fenómenos neurológicos, cardiovasculares, inmunitarios y metabólicos. ${ }^{9}$ Inicialmente se supuso que podía deberse a la presencia, en la vacuna entera, de una endotoxina capaz de alterar los niveles de insulina plasmática e inducir neutrofilia. Tanto en los estudios en seres humanos como en ratones se observó un aumento de la insulinemia, ${ }^{10,11}$ que si bien en los animales se correspondía con una

\section{TABLA 1. Definición de caso de episodio de hipotonía-hiporreactividad ${ }^{8}$}

- Episodio de inicio brusco ocurrido en las siguientes 48 horas a la administración de la vacuna en niños menores de 10 años.

- Deben estar presentes todas las siguientes manifestaciones:

- Hipotonía

- Escasa respuesta a los estímulos

- Palidez cutánea o cianosis

- No se considera episodio de hipotonía-hiporreactividad si existe causa conocida que pueda explicar estos signos, si existe urticaria o recuperación del color durante el episodio o si el paciente está simplemente dormido. 
reducción de la glucemia que podría justificar los síntomas de HHE, ${ }^{11}$ estos efectos sobre los niveles de glucosa en la sangre no se han podido demostrar en los seres humanos. ${ }^{10}$ Por otro lado, hay investigaciones que proponen una leucocitosis con neutrofilia, ${ }^{5,9}$ sin embargo, está también presente en sujetos vacunados que no han tenido HHE. En definitiva, no existen pruebas de laboratorio de utilidad para su confirmación y se trata de un proceso de diagnóstico clínico.

Debido a la elevada incidencia de efectos adversos neurológicos asociados a la administración de la vacuna preparada con el microorganismo inactivado, en la década de 1970 se impulsó el desarrollo de las vacunas acelulares. Estas pueden contener hasta cinco antígenos de Bordetella pertussis. ${ }^{12}$ Hay diferentes preparados de vacunas según el número de componentes antigénicos que lleven, pero todos contienen la toxina pertussis. Existe suficiente evidencia en cuanto a la similitud de eficacia entre las vacunas enteras y las acelulares. Se observó que las acelulares de tres y cinco componentes antigénicos son tan eficaces, o incluso más, que las vacunas enteras. ${ }^{12}$ Pero dicha eficacia no sólo depende del número de componentes, sino también de la cantidad de cada uno de ellos, la presencia de pertactina y sus métodos de producción. ${ }^{12}$ Las vacunas acelulares tienen una menor reactogenicidad, lo que da lugar a menor frecuencia de efectos adversos. ${ }^{1,3,12}$ Como no contienen endotoxina ni elevan la insulinemia, hay que considerar otros mecanismos que puedan explicar la menor frecuencia de complicaciones neurológicas.

Tanto en la administración de las vacunas acelulares como de las enteras, los HHE suelen observarse más a menudo después de las primeras dosis, lo que sugiere cierta protección desde la primera. No existe explicación para ello, pero deben influir las características de la vacuna y también fenómenos inmunitarios intrínsecos. ${ }^{5,13}$

En relación con la recurrencia, se observó una baja incidencia de repetición de HHE posadministración de DTPw, si bien se necesitan más datos para confirmar estos mismos hallazgos con la vacuna acelular. ${ }^{9}$

Respecto de la administración de dosis sucesivas de DTP en los niños que sufrieron efectos adversos, la resolución es controvertida. Actualmente, estos acontecimientos se consideran precauciones, pues no se ha demostrado que dichos episodios puedan ocasionar secuelas neurológicas a largo plazo. ${ }^{12}$ El Comité de
Enfermedades Infecciosas de la Academia Estadounidense de Pediatría ${ }^{14}$ recomienda basar la decisión de indicar o suspender la vacunación en la evaluación clínica de la reacción previa, la probabilidad de exposición del niño a la tos ferina, y los posibles beneficios y riesgos de la vacuna antitosferínica.

En definitiva, los HHE constituyen un fenómeno infrecuente como consecuencia de la administración de la vacuna contra la tos ferina, pero es fundamental que el personal sanitario sepa reconocerlos, sobre todo en las siguientes 48 horas a la vacunación. Aunque, en ocasiones, es difícil establecer una relación causal entre la administración de la vacuna y un efecto adverso, debe comunicarse cualquier evento sospechoso. ${ }^{15}$

\section{BIBLIOGRAFÍA}

1. Le Saux N, Barrowman NJ, Moore DL, Whiting S, et al. Decrease in hospital admissions for febrile seizures and reports of hypotonic-hyporesponsive episodes presenting to hospital emergency departments since switching to acellular pertussis vaccine in Canada: a report from IMPACT. Pediatrics 2003;112:e348.

2. Ministerio de Salud. Recomendaciones Nacionales de Vacunación Argentina 2012. [Consulta: 25 de octubre de 2012]. Disponible en: http://www.codeinep.org/RNVacunas_2012.pdf.

3. Pertussis vaccines: WHO position paper. Wkly Epidemiol Rec 2010;85:385-400.

4. Geier DA, Geier MR. An evaluation of serious neurological disorders following immunization: a comparison of whole-cell pertussis and acellular pertussis vaccines. Brain Dev 2004;26:296-300.

5. Buettcher M, Heininger U, Braun M, Bonhoeffer J, et al. Hypotonic-hyporesponsive episode (HHE) as an adverse event following immunization in early childhood: case definition and guidelines for data collection, analysis, and presentation. Vaccine 2007;25:5875-81.

6. Novy J, Catarino CB, Chinthapalli K, Smith SM, et al. Another cause of vaccine encephalopathy: a case of Angelman syndrome. Eur J Med Genet 2012;55:338-41.

7. Cherry JD, Karam AG. Hypotonic and hyporesponsive episodes after diptheria-tetanus-acellular pertussis vaccination. Pediatr Infect Dis J 2007;26:966-7.

8. Braun MM, Terracciano G, Salive ME, Blumberg DA, et al. Report of a US public health service workshop on hypotonic-hyporesponsive episode (HHE) after pertussis immunization. Pediatrics 1998;102:E52.

9. Gold MS. Hypotonic-hyporesponsive episodes following pertussis vaccination: a cause for concern? Drug Saf 2002;25:85-90.

10. Mink CM,Uhari M, Blumberg DA, Knip M, et al. Metabolic and hematologic effects and immune complex formation related to pertussis immunization. Pediatr Res 1990;27:3537.

11. Munoz JJ, Arai H, Bergman RK, Sadowski PL. Biological activities of crystalline pertussigen from Bordetella pertussis. Infect Immun 1981;33:820-6.

12. Jefferson T, Rudin M, DiPietrantonj C. Systematic review of the effects of pertussis vaccines in children. Vaccine. 2003;21:2003-14. 
13. Vermeer-de Bondt PE, Van der Maas NA. The effect of age and dose number on the risk of collapse (hypotonic-hyporesponsive episode) after pertussis vaccination. Pediatr Infect Dis J 2008;27:355-7.

14. Pickering LK, Baker CJ, Long SS, McMillan JA, Dirs. Pertussis. En American Academy of Pediatrics. Red Book: En- fermedades Infecciosas en Pediatría. $27^{\mathrm{a}}$ ed. Madrid: Editorial Médica Panamericana; 2007. Págs. 498-520.

15. McPherson P, Powell KR. Hypotonic-hyporesponsive episode in a 7-month-old infant after receipt of multiple vaccinations. Pediatr Infect Dis J 2005;24:1010-1. 